\title{
How do walking, standing, and resting influence transtibial amputee residual limb fluid volume?
}

\author{
Joan E. Sanders, PhD; ${ }^{1 *}$ John C. Cagle, BSE; ${ }^{1}$ Katheryn J. Allyn, CPO; ${ }^{1}$ Daniel S. Harrison, BS; ${ }^{1}$ Marcia A. \\ Ciol, PhD $^{2}$ \\ Departments of ${ }^{1}$ Bioengineering and ${ }^{2}$ Rehabilitation Medicine, University of Washington, Seattle, WA
}

\begin{abstract}
The purpose of this research was to determine how fluid volume changes in the residual limbs of people with transtibial amputation were affected by activity during test sessions with equal durations of resting, standing, and walking. Residual limb extracellular fluid volume was measured using biompedance analysis in 24 participants. Results showed that all subjects lost fluid volume during standing with equal weight-bearing, averaging a loss rate of $-0.4 \% / \mathrm{min}$ and a mean loss over the 25 min test session of $2.6 \%$ (standard deviation [SD] 1.1). Sixteen subjects gained limb fluid volume during walking (mean gain of 1.0\% [SD 2.5]), and fifteen gained fluid volume during rest (mean gain of $1.0 \%$ [SD 2.2]). Walking explained only $39.3 \%$ of the total session fluid volume change. There was a strong correlation between walk and rest fluid volume changes $(-0.81)$. Subjects with peripheral arterial disease experienced relatively high fluid volume gains during sitting but minimal changes or losses during sit-to-stand and stand-tosit transitioning. Healthy female subjects experienced high fluid volume changes during transitioning from sit-to-stand and stand-to-sit. The differences in fluid volume response among subjects suggest that volume accommodation technologies should be matched to the activity-dependent fluid transport characteristics of the individual prosthesis user.
\end{abstract}

Key words: activity, bioimpedance, compensation, diabetes, fluid transport, interface stress, peripheral arterial disease, prosthesis, prosthetic fit, volume accommodation.

\section{INTRODUCTION}

Residual limb volume change can cause socket fit problems for people who use prosthetic limbs. When the residual limb reduces in volume, the socket may become too loose, leading to excessive pistoning, gait instability, and possibly a fall or injury. When the residual limb increases in volume, the socket may become tight and uncomfortable. Excessive interface stresses may restrict vascular flow, denying cells of nutrients and inducing soft tissue injury. The volume a person loses or gains depends on several factors. Based on clinical experience, the most influential factors are considered to be the person's activity, health status, socket fit, and diet.

In this research study, we focused on better understanding the influence of one of these variables, type of activity, on residual limb fluid volume change. We collected data on health status, socket fit, and diet to ensure subjects were capable of completing the test protocol

\footnotetext{
Abbreviations: $\mathrm{ABI}=$ ankle-brachial index, $\mathrm{BMI}=$ body mass index, HBP = high blood pressure, ICF = intracellular fluid, $\mathrm{OBP}=$ orthostatic blood pressure, $\mathrm{PAD}=$ peripheral arterial disease, $\mathrm{SD}=$ standard deviation.

*Address all correspondence to Joan E. Sanders, PhD; Department of Bioengineering, University of Washington, Box 355061, William H. Foege Bldg, 3720 15th Ave NE, Seattle, WA 98195; 206-221-5872; fax: 206-616-2509. Email: jsanders@u.washington.edu http://dx.doi.org/10.1682/JRRD.2013.04.0085
} 
without injury but did not control those variables. People using prostheses typically comment that when they are very active they experience limb volume loss and need to add socks to compensate. We would therefore expect residual limb fluid volume loss to be much greater during walking than during rest. Researchers have reported that subjects using total contact suction sockets lost an average of 3.7 percent of their residual limb volume after 30 min of continuous walking on a treadmill [1]. The same researchers reported that subjects using elevated vacuum sockets gained 6.5 percent volume from before to after $30 \mathrm{~min}$ of continuous treadmill walking [1]. In case studies, our research group found that during $5 \mathrm{~min}$ of treadmill walking, subjects with vascular health problems lost more fluid volume than subjects without vascular problems [2]. In a separate study, in a group of 30 participants doffing their prosthesis right after activity and sitting quietly, we found that residual limb fluid volume in 23 of them either maximized or plateaued within $10 \mathrm{~min}$, while fluid volume in 7 of them was still increasing at the end of $10 \mathrm{~min}$ [3]. When subjects doffed their prosthesis after walking, fluid volume recovery averaged $4.9 \pm 2.1$ percent (mean \pm standard deviation $[\mathrm{SD}]$ ) over $30 \mathrm{~min}$ [4]. Leaving the prosthesis donned induced a mean \pm SD fluid volume loss of $2.2 \pm 1.2$ percent.

The purpose of this research was to compare limb fluid volume changes during different activities (resting, standing, walking) in subjects with transtibial amputation. If different activities induced different fluid volume changes, then insight useful toward clinical prosthetic fitting would be achieved. Potentially, we could design technologies to operate during specific activities to counteract excessive fluid volume losses. Data from an activity monitor mounted to the prosthesis could be used to adjust, either through instruction to the patient or automatically, volume accommodation systems (e.g., elevated vacuum, fluid-filled bladders, socket-shape adjustment devices, sock addition/removal).

The following questions were addressed in this research: During test sessions with equal durations of resting, standing, and walking, do subjects lose the most fluid volume during walking? How do fluid volume changes during walking compare with those during standing and resting? We also investigated how much variability there was in data across subjects. Did all subjects lose fluid volume during walking and standing and gain fluid volume during resting? Did all subjects who lost fluid volume during walking lose fluid volume over the entire test session? Was there variability in the time course of recovery during resting? Finally, we explored whether variability in fluid volume change data was affected by subject characteristics including sex, peripheral arterial disease (PAD), high blood pressure (HBP), and diabetes.

\section{METHODS}

Human subject volunteers were included in this study if they had a transtibial amputation at least 6 mo prior and were using a definitive prosthetic limb at least $5 \mathrm{~h} / \mathrm{d}$. The prosthesis was required to fit properly as deemed by the research prosthetist. Subjects needed to be capable of $90 \mathrm{~s}$ of continuous treadmill walking at a self-selected walking speed as well as $90 \mathrm{~s}$ of continuous standing. Residual limb length needed to be at least $9 \mathrm{~cm}$ to allow electrodes to be properly spaced when placed on the residual limb. Subjects were not included if they had current skin breakdown, were unable to wear a prosthesis for at least $1.5 \mathrm{~h}$ continuously, and were unable to shift from standing to supine posture within $30 \mathrm{~s}$ (for vascular tests). Subjects were asked to consume a normal breakfast but to refrain from consuming caffeine and alcohol before coming to the laboratory on test days. All study procedures were approved by a University of Washington Institutional Review Board, and informed consent was obtained before any study procedures were initiated.

On a separate day before bioimpedance testing but not more than 12 mo prior, using techniques described in detail previously [5], we tested the subjects for presence of HBP (orthostatic blood pressure [OBP]), and PAD (segmental limb pressures, ankle-brachial index $[\mathrm{ABI}]$ ). We used an electronic blood pressure measurement unit (HEM-775, Omron; Kyoto, Japan) for OBP testing and a commercial segmental limb pressure measurement system (TD312 Cuff Inflator, MV10 Manifold Selector, and SC12 and SC10 cuffs, Hokanson; Bellevue, Washington) for segmental limb pressures and ABI assessment. Collected data were interpreted by a practicing endocrinologist using standard clinical procedures [6-7].

We modified a commercial bioimpedance analyzer (XiTRON Hydra 4200, Impedimed; San Diego, California) to measure residual limb fluid volume on people with limb amputation. Electrical current between 100 and $700 \mu \mathrm{A}$ was injected at 50 frequencies between $5 \mathrm{kHz}$ and $1 \mathrm{MHz}$ through current-injection electrodes on proximal and distal aspects of the residual limb (Figure 1). 


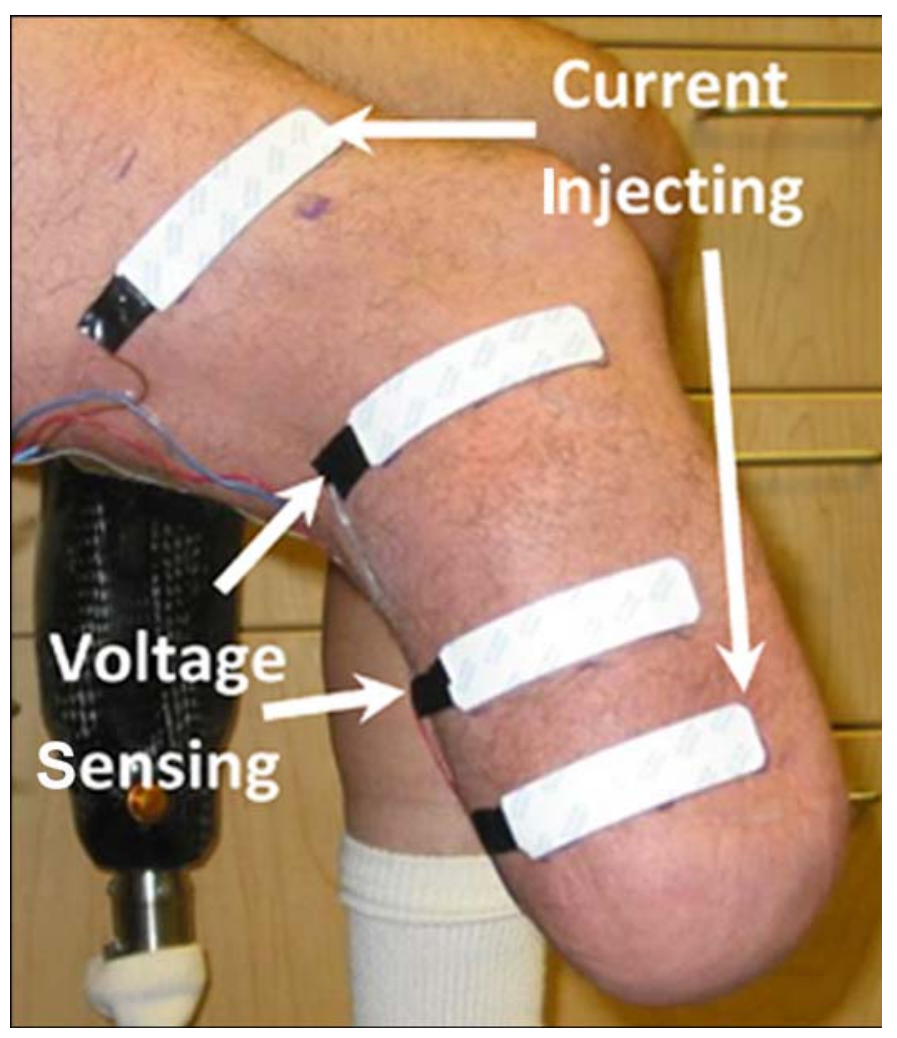

Figure 1.

Residual limb instrumented with electrodes for bioimpedance testing.

Voltage was sensed with voltage-sensing electrodes positioned between the two current-injecting electrodes. The current and voltage signals were demodulated within the XiTRON unit to calculate magnitude and phase difference for each frequency. The sampling rate of the XiTRON instrument was approximately $1 \mathrm{~Hz}$.

Electrodes provided by the manufacturer were used (XiTRON, $77 \times 20 \mathrm{~mm}$ contact surface, $0.81 \mathrm{~mm}$ thickness). Care was taken to fabricate a stable electrode-toleadwire connection [5]. A thin layer of ultrasonic coupling gel (Couplant D, GE Panametrics; West Chester, Ohio) was placed on the underside of each electrode. Wires extending from the electrodes proximally to the XiTRON were strain-relieved using Tegaderm (3M; St. Paul, Minnesota). Outside the socket, a custom connector with gold-plated pins (WPI Viking, Cooper Interconnect; Chelsea, Massachusetts) was used to connect the four electrodes to a coaxial cable that attached to the XiTRON unit with a robust connector (MS3116F106S, Burndy; Manchester, New Hampshire). Care was taken to ensure that the presence of the wires did not cause air channels to form, which would have allowed air to escape from the limb-socket interface and induce a loss of suspension. The peak-to-peak fluctuation in the bioimpedance signal while the subject stood with equal weight-bearing was less than 0.2 percent of the limb fluid volume.

After the subject arrived at the laboratory, we recorded the subject's mass and height wearing his or her prosthesis. Body mass index (BMI) was calculated as mass in kilograms divided by the square of height in square meters. No correction was made to BMI for amputation. Subjects with a BMI greater than 30 were considered obese, and those with a BMI between 25 and 30 were considered overweight. The subject sat for 10 to $15 \mathrm{~min}$ to achieve a homeostatic condition and answered questions about presence of diabetes or other illness and recent changes to his or her prosthesis. Then, the subject doffed the prosthesis and the research practitioner inspected the subject's residual limb for soft tissue injury. She also evaluated whether socket fit was acceptable for regular prosthesis use. If breakdown was present or socket fit was unacceptable, then the test session was terminated and the subject was referred to his or her regular practitioner. Otherwise, the session was continued and sites where electrodes were to be placed were cleaned (Tracer Prep, 3M). Electrodes were placed on the limb such that all electrodes were parallel with each other. The proximal voltage-sensing electrode was placed at the level of the patellar tendon on the posterior lateral surface of the limb proximal of the fibular head. The distal current-injecting electrode was placed as far distally as possible but still on the cylindrical portion of the residual limb. The distal voltage-sensing electrode was placed at least $3 \mathrm{~cm}$ proximal of the distal current-injecting electrode. The proximal current-injecting electrode was placed an average of $9 \mathrm{~cm}$ proximal to the proximal voltage electrode, outside of the socket under the proximal end of the elastomeric liner or sleeve suspension.

We initiated continuous bioimpedance data collection. Bioimpedance data were viewed in real time (1 to $3 \mathrm{~s}$ delay) using custom MATLAB (MathWorks; Natick, Massachusetts) code that implemented a Cole model [8], similar to that used in the XiTRON postprocessing program [9], so that we could identify set-up problems if they existed.

The subject donned the prosthesis and rested in a chair for $90 \mathrm{~s}$ (REST). Care was taken to ensure a proper sitting posture during all rest periods. A proper sitting 
posture was characterized by relaxed legs and a knee flexion angle of approximately $130^{\circ}$. Following the $90 \mathrm{~s}$ REST, the subject was asked to stand for $90 \mathrm{~s}$ with equal weight-bearing (STAND) with the prosthetic limb supported by an electronic scale (349KLX Health-O-Meter, Pelstar; Alsip, Illinois) embedded within a short platform so that it was flush with the surface. If the subject's weight-bearing on the prosthesis deviated by more than 10 percent of half the body weight, the subject was asked to shift his or her weight accordingly. Then, the subject moved onto a treadmill and walked for $90 \mathrm{~s}$ at a selfselected walking speed (WALK). The same speed was used for all WALK cycles in a session for each subject. The subject then returned to the scale to stand under equal weight-bearing for approximately $10 \mathrm{~s}$ and then sat down on the chair. The cycle of 90 s REST, 90 s STAND, $90 \mathrm{~s}$ WALK, and $10 \mathrm{~s}$ STAND was then repeated four additional times. The five cycles took less than $25 \mathrm{~min}$ to complete. At the conclusion, the subject sat down and doffed his or her prosthesis. Bioimpedance data collection was terminated and the electrodes were removed.

Demodulated data stored to disk from the XiTRON were converted to extracellular and intracellular fluid (ICF) impedances using a Cole modeling strategy [8]. An anatomical limb model was used to calculate extracellular fluid volume from the Cole model results and limb dimension measurements [10]. We found that some subjects required part of the first WALK cycle to adjust to the treadmill and accomplish a repeatable gait. Thus, we did not include the first REST/STAND/WALK cycle in analysis and instead started analysis from the brief stand after completion of the first REST/STAND/WALK cycle. Limb fluid volume during this brief stand was defined as the reference limb fluid volume for the trial. The beginning and the end of each REST, STAND, and WALK phase within each of the four subsequent cycles were identified and labeled. Example data for two and a half cycles are shown in Figure 2.

Fluid volume changes during each phase (REST, STAND, and WALK) of each of the last four REST/ STAND/WALK cycles were calculated. The REST change in each cycle was calculated as the fluid volume at the beginning of the subsequent STAND minus that during the previous brief stand after the previous WALK. The STAND change was the fluid volume at the end of the $90 \mathrm{~s}$ STAND minus that at the beginning of the $90 \mathrm{~s}$ STAND. The WALK change was calculated as the fluid volume during the brief stand after WALK minus that at

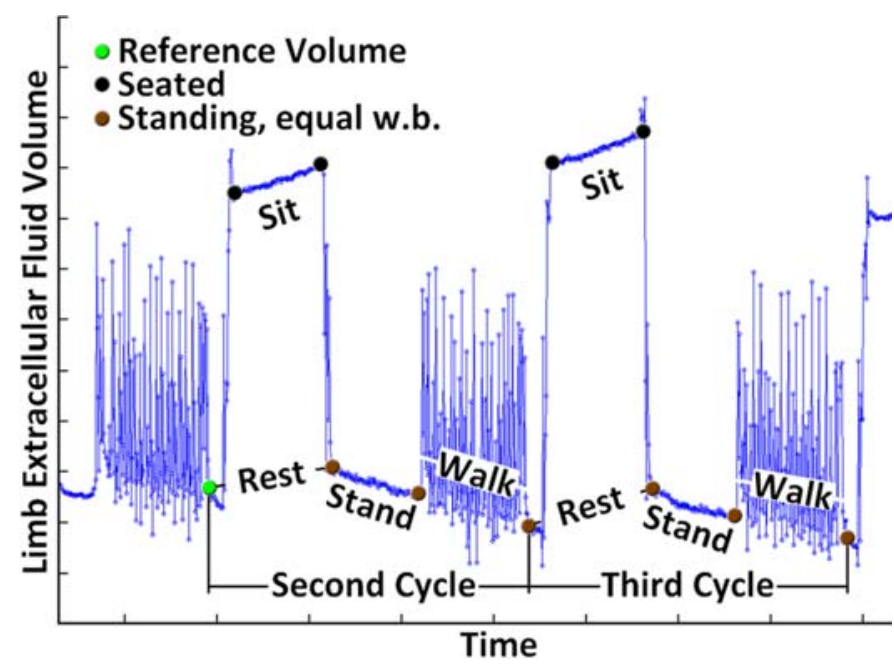

Figure 2.

Definitions of types of activities. REST, STAND, and WALK activities were conducted. REST includes both SIT and TRANSITION from walk-to-sit and sit-to-walk. Limb extracellular fluid volume is in arbitrary units in this figure. w.b. = weight bearing.

the end of the immediately prior STAND. Thus, only data collected during standing with equal weight-bearing were used to calculate fluid volume changes during the three phases (REST, STAND, and WALK). We calculated coefficient of variation (Var) for REST, STAND, and WALK using-

$$
\operatorname{Var}_{\text {Avg }}=\frac{\sum_{i=\text { Subject } \operatorname{Var}_{i}}^{n}}{n_{\text {subjects }}}
$$

and

Subject $\operatorname{Var}_{i}=\frac{\mathrm{SD}(\text { Cycle } 2+\text { Cycle } 3+\text { Cycles } 4+\text { Cycle } 5)}{\text { Mean }(\text { Cycle } 2+\text { Cycle } 3+\text { Cycle } 4+\text { Cycle } 5)}$.

We also calculated fluid volume changes during the TRANSITION (stand-to-sit plus sit-to-stand) and SIT parts of the REST phases. We reasoned that subjects with PAD would experience relatively slow fluid movement in and out of their residual limb during resting. Therefore, they would be expected to have low fluid volume changes during TRANSITION and high fluid volume changes during SIT, a low TRANSITION-SIT difference. Subjects without PAD were expected to have fast fluid transport thus would be expected to experience high fluid volume 
changes during TRANSITION and low fluid volume changes during SIT, a high TRANSITION-SIT difference.

SIT fluid volume change was calculated as the fluid at the end of the SIT phase minus that at the beginning of the SIT phase (Figure 2). We quantified TRANSITION fluid volume changes as any changes during REST that were not within SIT. TRANSITION was the SIT magnitude subtracted from the REST magnitude. Thus, TRANSITION was the sum of the fluid volume change both from sitting down and standing up. We did not separate standto-sit from sit-to-stand fluid volume change because of knee flexion differences between sitting and standing.

The fluid volume changes during the last four cycles of each phase (REST, STAND, and WALK) as well as those for the subgroups of REST, i.e., TRANSITION and SIT, were summed for each subject over the test session. We summed the data for the separate phases so that we could compare results with total fluid volume changes over the session (TOTAL). TOTAL fluid volume change was defined as the fluid volume during the brief stand after the last REST/STAND/WALK minus the reference fluid volume for the trial (i.e., fluid volume measured during the brief stand after the first REST/STAND/ WALK cycle). All data were expressed as a percentage change relative to the reference fluid volume for the trial.

To compare percent changes between two activities, we used paired $t$-tests with bootstrap robust estimation of the standard errors; thus, assumption of normal distribution of the paired differences was not required. Pearson correlation was used to assess the association between outcomes. To assess whether the percent WALK fluid volume change would be a predictor of the percent TOTAL change, we performed a linear regression analysis with the latter as a response variable and the former as an explanatory variable. To explore whether the distributions of fluid volume changes were different for certain subject characteristics, such as sex and presence of PAD, we conducted nonparametric Mann-Whitney tests. Significance level for all tests was 0.05 . However, due to the small sample size and large variation of the data, the results must be interpreted as preliminary and exploratory.

In addition to statistical analyses to address the central research questions, we conducted an exploratory analysis to identify relationships between fluid volume changes and subject characteristics. Subject characteristics considered included sex, PAD, HBP, and diabetes. We could not explore the influence of socket type, suspension, and sockply since the sizes of some categories of those variables were very small (a single person, in some cases - see Table 1) and not sufficient to provide insight in how fluid volume was associated with these variables.

\section{RESULTS}

A total of 26 volunteers participated in the study. However, data from two subjects were not included in

Table 1.

Subject and prosthesis characteristics.

\begin{tabular}{|c|c|c|c|c|}
\hline Characteristic & Range & Mean \pm SD & Median & No. Participants \\
\hline \multicolumn{5}{|l|}{ Subjects } \\
\hline Age (yr) & $23.3-72.3$ & $51.8 \pm 13.3$ & 53.8 & - \\
\hline Height (m) & $162.6-233.7$ & $179.4 \pm 15.5$ & 181.6 & - \\
\hline Mass (kg) & $54.7-124.5$ & $88.8 \pm 20.0$ & 88.0 & - \\
\hline BMI $\left(\mathrm{kg} / \mathrm{m}^{2}\right)$ & $14.9-44.0$ & $27.7 \pm 6.2$ & 27.3 & - \\
\hline Time Since Amputation (yr) & $0.5-49.5$ & $11.7 \pm 13.2$ & 8.2 & - \\
\hline Male/Female & - & - & - & $17 / 7$ \\
\hline Not Overweight/Overweight/Overweight and Obese & - & - & - & $3 / 14 / 7$ \\
\hline Smoker & - & - & - & 7 of 24 \\
\hline Presence of PAD & - & - & - & 11 of 24 \\
\hline Presence of HBP & - & - & - & 13 of 24 \\
\hline Presence of Diabetes & - & - & - & 8 of 24 \\
\hline \multicolumn{5}{|l|}{ Prostheses } \\
\hline Socket Design & - & - & - & 23 РTB; 1 PTB-suction \\
\hline Liner Type & - & - & - & $\begin{array}{l}9 \text { Alpha; } 9 \text { Iceross; } 4 \text { Pelite; } 1 \text { ALPS; } \\
1 \text { custom elastomer }\end{array}$ \\
\hline Suspension & - & - & - & 18 pin lock; 3 suction; 3 sleeve \\
\hline Sock Ply & - & - & - & $80-1$ ply; $82-3$ ply; $74-10$ ply; 113 ply \\
\hline
\end{tabular}


analysis because their residual limb lengths were outside of the calibration range acceptable for use of the bioimpedance instrument. Data from the remaining 24 subjects are presented here. Subject and socket characteristics are listed in Table 1.

\section{Comparison of REST, STAND, and WALK Fluid Volume Changes}

Analysis of fluid volume changes over the test session for the different activities (REST, STAND, and WALK) showed that the highest mean fluid volume loss was during STAND, with a mean \pm SD of $2.6 \pm 1.1$ percent loss over the test session (Table 2). On average, subjects gained fluid volume during WALK and REST, with a mean \pm SD limb fluid volume increase of $1.0 \pm 2.5$ percent during WALK and $1.0 \pm 2.2$ percent during REST. Fluid volume changes during STAND were significantly different from those during WALK $(p=0.001)$ and from those during REST $(p=0.001)$. REST and WALK fluid volume changes were not significantly different from each other $(p=0.91)$.

Limb fluid volume changes for each subject were relatively consistent across the four cycles. Average coefficients of variation across the four cycles were 1.3 for REST, 0.2 for STAND, and 0.7 for WALK.

\section{Variability and its Dependence on Subject Characteristics}

All participants lost fluid volume during STAND. We calculated the rate of fluid volume loss within the STAND periods to investigate how rapidly fluid volume losses occurred. The mean $\pm \mathrm{SD}$ rate of change was $-0.4 \pm$ $0.2 \mathrm{percent} / \mathrm{min}$.

Not all participants lost fluid volume during WALK. Eight participants lost fluid volume during WALK, while sixteen gained. Not all participants gained fluid volume during REST. Fifteen participants gained fluid volume during REST, while nine lost. All except three subjects experi-

Table 2.

Percentage fluid volume changes relative to reference fluid volume during different activities for all subjects.

\begin{tabular}{llcc}
\hline \multicolumn{1}{c}{ Activity } & \multicolumn{1}{c}{ Range } & Mean \pm SD & Median \\
\hline REST (TRANS+SIT) & -1.9 to 5.7 & $1.0 \pm 2.2$ & 0.4 \\
STAND & -5.4 to -0.7 & $-2.6 \pm 1.1$ & -2.6 \\
WALK & -4.9 to 5.7 & $1.0 \pm 2.5$ & 1.3 \\
\hline TOTAL & -2.7 to 2.4 & $-0.6 \pm 1.3$ & -0.9 \\
\hline SD $=$ standard deviation, TRANS $=$ transition.
\end{tabular}

enced greater fluid volume losses during STAND than during WALK. All except one subject experienced greater fluid volume losses during STAND than during REST.

Table 3 shows the correlations between the various activities and with the percent total fluid volume change over the test session (TOTAL). The highest absolute value correlation between activities was for WALK and REST (0.81), followed by STAND and REST (0.41). A high absolute value correlation, close to 1.00 , indicates a strong relationship between the variables. Thus, in general, subjects who lost fluid volume during WALK gained fluid volume during REST. WALK was the only activity for which there was a statistically significant correlation with TOTAL (0.63). Figure 3 shows the percentage TOTAL fluid volume change by the percentage WALK fluid volume change. Only 6 individuals (25\%) lost fluid volume for WALK and TOTAL (quadrant (d)), while 13 gained in WALK but lost during the entire session (54.2\%, quadrant (a)). However, linear regression analysis showed that percent WALK fluid volume change explained only 39.3 percent of the variation of TOTAL. The analysis of residuals showed that the model did not fit the data well. Therefore, percent WALK fluid volume change was not a good predictor of TOTAL fluid volume change.

To assess whether percent volume change was associated with sex, PAD, HBP, and diabetes, we compared the distribution of percent fluid volume changes for all three activities. There were no statistically significant differences in distribution for males versus females for WALK $(p=0.66)$, STAND $(p=0.26)$, REST $(p=0.32)$, and TOTAL $(p=0.09)$; for individuals with and without PAD for WALK $(p=0.91)$, STAND $(p=0.25)$, REST $(p=$ $0.49)$, and TOTAL $(p=0.57)$; and for individuals with and without diabetes for WALK $(p=0.93)$, STAND $(p=0.19)$, $\operatorname{REST}(p=0.61)$, and TOTAL $(p>0.99)$. For presence of HBP, there were no statistically significant differences in distributions of percentage fluid volume change between individuals with and without HBP for WALK $(p=0.61)$, REST $(p=0.46)$, and TOTAL $(p=0.30)$, but the two groups were statistically different for STAND $(p=0.02)$,

\section{Table 3.}

Correlations of limb fluid volume changes between REST, STAND, WALK, and TOTAL. Boldface indicates statistically significant results at 0.05 level.

\begin{tabular}{lccc}
\hline \multicolumn{1}{c}{ Activity } & REST & STAND & WALK \\
\hline STAND & $\mathbf{- 0 . 4 1}$ & - & - \\
WALK & $\mathbf{- 0 . 8 1}$ & 0.03 & - \\
TOTAL & 0.26 & 0.22 & $\mathbf{0 . 6 3}$ \\
\hline \hline
\end{tabular}




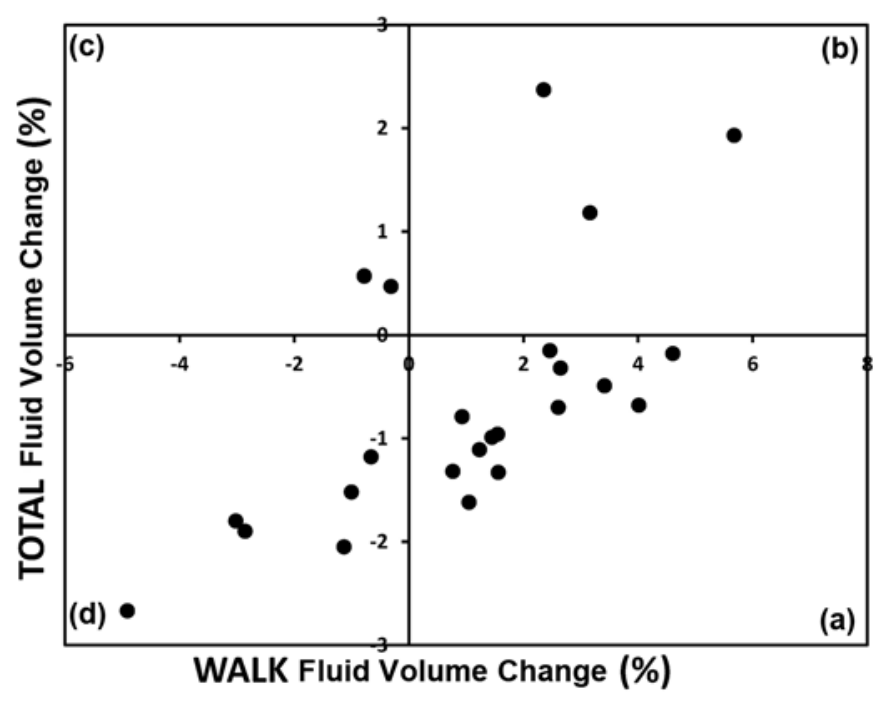

Figure 3.

Percentage TOTAL fluid volume change by WALK fluid volume change. Correlation between TOTAL fluid volume change and WALK fluid volume change was 0.63. However, WALK explained only 39.3 percent of TOTAL, thus was not good predictor of TOTAL. Most subjects either gained fluid volume during walking and lost during rest (quadrant (a), 13 subjects) or lost fluid volume during both walk and rest (quadrant (d), 6 subjects).

where loss of fluid volume was larger among people without HBP (median $=-2.8$ ) when compared with people with HBP (median $=-1.9$ ). For smoking status, no statistically significant differences were found for WALK $(p=$ $0.58)$, STAND $(p=0.29)$, REST $(p=0.95)$, and TOTAL $(p=0.90)$. Tests of hypothesis were not performed for BMI category, socket type, suspension, and sock-ply because there were too few individuals in some of the categories of those characteristics and the variation made statistical tests invalid under those circumstances.

Median values for TRANSITION were not statistically different for sex $(p=0.17)$ HBP $(p=0.42)$, and diabetes $(p=0.09)$, but were statistically different for $\operatorname{PAD}(p=0.02)$. Median values for SIT were not statistically different for sex $(p=0.46)$ or $\operatorname{HBP}(p=1.0)$, but were statistically different for PAD $(p<0.001)$ and diabetes $(p=0.02)$.

\section{Time Course of Recovery}

There was considerable variability in TRANSITION and SIT fluid volume changes among subjects (Table 4). Eleven subjects lost fluid volume during TRANSITION, while thirteen gained. Five subjects lost fluid volume
Table 4.

Percentage fluid volume changes relative to reference fluid volume during TRANSITION and SIT for all subjects.

\begin{tabular}{lccc}
\multicolumn{1}{c}{ Activity } & \multicolumn{1}{c}{ Range } & Mean \pm SD & Median \\
\hline TRANSITION & -5.1 to 5.3 & $-0.2 \pm 2.5$ & 0.0 \\
SIT & -1.4 to 4.9 & $1.1 \pm 1.6$ & 0.7 \\
\hline SD $=$ standard deviation. & & \\
\hline \hline
\end{tabular}

during SIT, while nineteen gained. There was thus much variability in the time course of fluid volume recovery during REST.

Extending from the previous results, we conducted an exploratory analysis to determine whether subjects with common TRANSITION/SIT/WALK fluid volume change patterns had common characteristics (we did not include STAND results in the exploratory analysis since all subjects lost fluid volume during STAND). If subjects with common TRANSITION/SIT/WALK fluid volume change patterns had common characteristics, then potentially subject characteristics could be used to predict fluid volume change patterns, information useful toward prosthetic design and fitting. We divided subjects into those that lost fluid volume during WALK and those that gained. The basis for selecting direction of WALK fluid volume change as an initial delineator of subjects into subgroups was our prior experience that subjects who lost fluid volume during walk typically had health problems [2]. Then, within each of those two collections of participants, we ordered subject data from low to high TRANSITION-SIT differences. We expected subjects with low TRANSITION-SIT values to experience slow fluid transport and thus to be in poor health. Four groups emerged (Figure 4):

- Group 1: Subjects who lost fluid volume during WALK, gained during SIT, had relatively little fluid volume change during TRANSITION, and thus had low TRANSITION-SIT values. Three of the four subjects had PAD.

- Group 2: Subjects who lost fluid volume during WALK, had relatively little change during SIT, and gained fluid volume during TRANSITION. Three of the four subjects were female, and none had PAD.

- Group 3: Subjects who gained fluid volume during WALK and SIT but lost much fluid volume during TRANSITION. Six of the six subjects had PAD.

- Group 4: Subjects who gained fluid volume during WALK but experienced relatively little change during SIT and TRANSITION. Two out of ten subjects had PAD. 


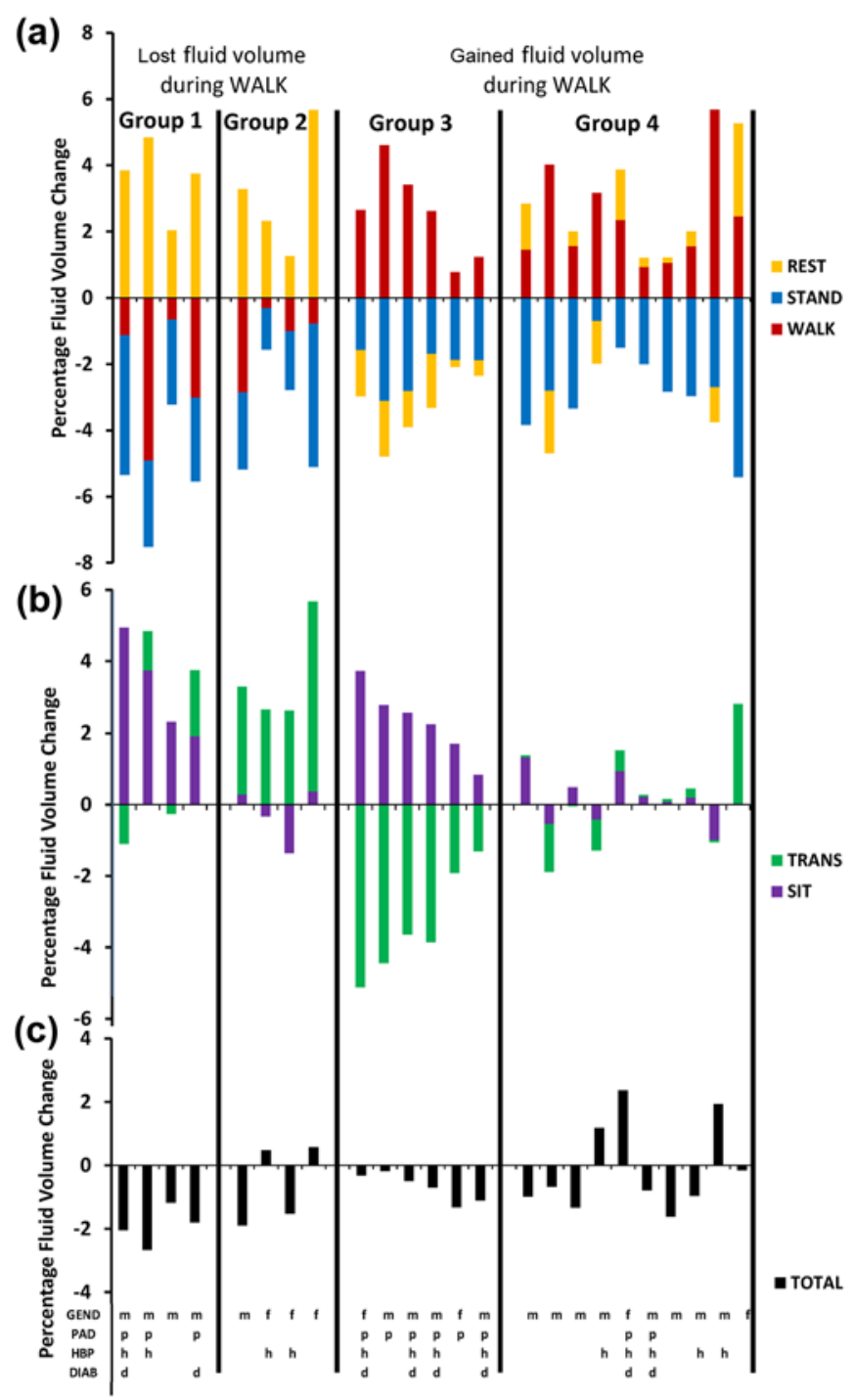

Figure 4.

Percentage fluid volume change for different activities and subject characteristics: (a) REST, STAND, and WALK; (b) TRANSITION (TRANS) and SIT; and (c) TOTAL. Subject data are ordered left-to-right from low to high TRANSITION-SIT percentage fluid volume differences for subjects who lost fluid volume during WALK and for subjects who gained fluid volume during WALK. Third subject from left was on lifetime antibiotics.

\section{DISCUSSION}

Measurement of fluid volume changes during different activities in people with limb amputation may provide practitioners with insight useful toward prosthetic design and toward counseling their patients on when to expect fluid volume changes in their residual limb. The data may also provide information useful toward the design of control strategies to adjust prostheses to accommodate limb fluid volume fluctuations based upon the activity the prosthesis user is currently conducting or recently conducted.

We considered sources of error and their potential impact on the data. We used a four-electrode array configuration to minimize impact of skin impedance on collected data. The impedance of skin is high, and bone is relatively nonconductive. Muscle (and fluid within the muscle fascia compartments) is highly conductive. Thus, bioimpedance analysis measures fluid volume primarily of muscle and surrounding soft tissues between the skin and underlying bone. Our experience is that bone serves as an effective barrier isolating the anterior from the posterior region of the residual limb. Thus, in the present study, the measurements are from the lateral and posterior muscle regions. It should be noted that the anterior region and the very distal region of the residual limb were not monitored in this study. This constraint is a limitation of electrode placement and the number of channels available on the XiTRON unit.

It is possible to use Cole modeling to calculate ICF impedance [11]. However, because of excessive noise at high current-injection frequencies, we considered ICF measurements on amputees from the XiTRON instrument inaccurate and did not report them here. Intracellular measurements are of interest, in part because they may provide better insight into blood volume changes in the residual limb. As bioimpedance technology for residual limb assessment improves, accurate ICF volume measurement should be possible.

A challenge in using biompedance analysis on people with limb loss wearing prosthetic limbs is that changes in skin strain may affect the distance between voltage-sensing electrodes and distort the measurement. To avoid this source of data misinterpretation, we used in our analysis of REST, STAND, and WALK fluid volume changes data collected while the subject was in a consistent posturestanding with equal weight-bearing. This strategy ensured consistent knee flexion from trial to trial, a variable that might otherwise affect skin strains between the voltagesensing electrodes and thus affect interpretation of the measurement. Similarly, SIT fluid volume change was calculated with the subject in a consistent posture from the beginning to the end of each SIT period, avoiding the effect of change in knee flexion on the results. 


\section{Comparison of REST, STAND, and WALK Fluid Volume Changes}

The result that STAND was the dominant source of fluid volume loss is contrary to clinical expectation. WALK would be expected to cause the greatest fluid volume loss. However, the result that most of the fluid volume losses over the session occurred during STAND is consistent with physical and physiologic constraints experienced by a residual limb within a prosthetic socket. During STAND, because the limb is enclosed by the socket, pressures applied at the limb-socket interface to support weight-bearing increase the pressure within the interstitial space inside the residual limb. This increased interstitial fluid pressure causes interstitial-to-venous fluid transport to dominate over arterial-to-interstitial fluid transport, illustrated conceptually in Figure 5. There is no compensatory physiological mechanism during STAND, for

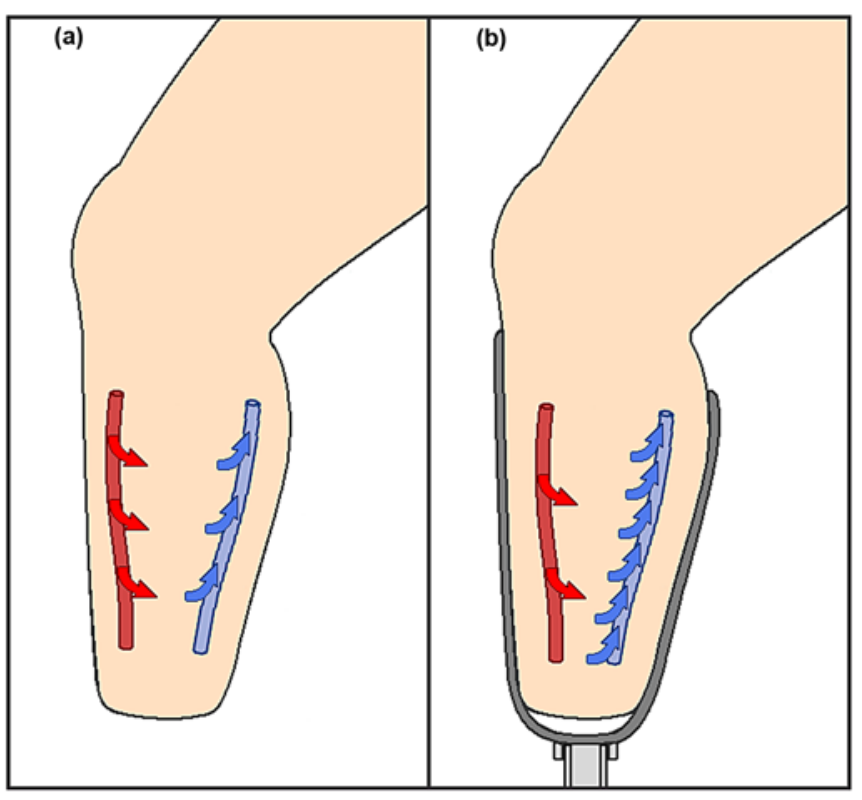

Figure 5.

Conceptual illustration of interstitial fluid transport balance/ imbalance in residual limb. Red arrows = volume flow of transport from arteries into interstitium. Blue arrows = volume transport from interstitium into veins. (a) During resting with socket doffed. Fluid volume transport is in balance. (b) During STAND. Fluid volume transport is out of balance. More fluid is entering veins and exiting residual limb than is moving across arteries and entering residual limb, resulting in overall tissue fluid volume loss and reduction in residual limb volume. example no intermittent pressure release, that counters this imbalance. The result for all participants is thus a gradual fluid volume loss during STAND.

\section{Variability and its Dependence on Subject Characteristics}

For the 16 of 24 subjects who gained fluid volume during WALK, we suspect several possible mechanisms were at work during WALK to accomplish fluid volume gains: muscle in the residual limb actively pumping fluid into the residual limb during swing phase; elevated arterial pressure during activity, increasing arterial fluid drive; and proximal displacement of the limb in the socket during swing phase to release pressures intermittently and thus facilitate fluid volume return. It is also possible that the immediately prior STAND period in the protocol temporarily dehydrated the residual limb and served to accentuate the subsequent WALK fluid volume gains.

We expected based upon clinical experience that WALK would have the greatest impact on TOTAL fluid volume change. However, our results showed that WALK explained only 39.3 percent of TOTAL. Our results suggests that clinicians need to consider other activities besides walking when helping a patient understand what causes limb fluid volume change and predict their daily volume fluctuation.

The strong linear correlation between REST and WALK $(-0.81)$ in the present study suggests that to some extent we can predict that a person who loses a lot of fluid volume during WALK will gain a lot during REST and vice versa. Subject education about doffing or releasing the socket when a long sitting interval is anticipated may be helpful toward stabilizing limb fluid volume.

\section{Time Course of Recovery}

After finding that 16 of 24 subjects gained fluid volume during WALK, we investigated whether the 8 subjects who lost fluid volume during WALK all had PAD. We found that less than half of them (3 of 8 ) did (Figure 4). The four subjects who lost fluid volume during WALK but were healthy (Group 2) did show one noticeable difference compared with the four subjects with PAD or on lifetime antibiotics who lost fluid volume during WALK (Group 1). They experienced high TRANSITION fluid volume increases, thus quickly moved fluid into and out of the residual limb upon sitting down and standing up. A large study needs to be conducted to determine whether there is a link between presence of 
fluid volume loss during WALK, subject health, and rate of fluid volume change during REST.

The difference in behavior during REST between participants in Group 2 and Group 1 is relevant to prosthetic fitting because it suggests different accommodation strategies should be used to facilitate fluid volume recovery during REST. We did not test any accommodation strategies in the present study; thus at this point, we can only hypothesize that the following approaches may be effective for the different groups: using a socket-release strategy in which the volume of the socket is temporarily enlarged while the subject rests, either through doffing or an automated socket-release mechanism, participants in Group 1 (Figure 4) would be expected to need a relatively long release interval to facilitate fluid volume recovery. Subjects in Group 2 (Figure 4) would be expected to require only a short release interval. Too long a release interval for Group 2 subjects might create an edematous limb, making it difficult to re-don the prosthesis when returning to weight-bearing. Alternatively, Group 2 subjects might instead be able to retard fluid volume loss using suction sockets or elevated vacuum technology. Because they have the capability to recover limb fluid volume quickly, those technologies might accentuate their limb fluid volume recovery during swing phase. Use of these different strategies needs to undergo thorough research investigation to prove or disprove these hypotheses.

Though most participants compensated for fluid volume loss during STAND by increasing fluid volume during WALK, some of them experienced so much fluid volume loss during REST that their overall fluid volume change was negative (Group 3). All Group 3 participants (6 of 6) had PAD as shown in Figure 4. Interestingly, Group 3 subjects lost considerable volume during TRANSITION, not during SIT. This result suggests that fluid that was gained during SIT was easily displaced out of the limb upon rising for the subsequent STAND. Because of the quick expulsion of fluid upon standing, we suspect that the fluid was primarily blood within the vascular network as opposed to fluid within the interstitial space. We were not able to verify this suspicion because there was no available device to measure blood volume in the residual limb with sufficient accuracy to meet the measurement need. Bioimpedance analysis has the potential to meet the need since it is capable of ICF volume assessment [11]. ICF volume assessment might help differentiate blood from other residual limb fluid. We did not include ICF analysis in the present study because of excessive noise in the ICF signal.

For Group 3 subjects, an effective accommodation strategy might be one that reduced TRANSITION fluid volume loss, possibly through a quick-release mechanism that relieved interface pressures immediately upon sitting, combined with prolonged socket release during resting so as to maximize SIT fluid recovery. Again, use of this strategy needs to undergo thorough research investigation to further test this hypothesis.

Based on our experience using biompedance analysis on people with comorbidities, the two outlier subjects with PAD in Figure 4 (5th and 6th from right) who did not fall within Groups 1 or 3 like the other subjects with PAD may have also had venous insufficiency, offsetting reduced arterial-to-interstitial transport with reduced interstitial-to-venous transport. Presence of venous insufficiency is difficult to evaluate because it often is not systemic but instead localized, induced by amputation surgery, for example. A subject might not demonstrate presence of venous insufficiency in the contralateral limb using standard test methods (ambulatory strain gauge plethysmography [ASGP] testing [12]) but may still have venous insufficiency in the residual limb and vice versa.

For subjects in Group 4 (Figure 4), fluid volume changes over the test session were dominated by differences between STAND and WALK fluid volume changes. If these subjects spent more time walking and less time standing, possibly their daily fluid volume loss would decrease. This hypothesis needs to be tested in research investigations. If the hypothesis were proven, then these subjects would benefit from practitioner education on this topic so as to help them better understand, predict, and accommodate their daily limb fluid volume change.

We observed that subjects with high TRANSITION values (Group 2 and rightmost subject in Group 4, Figure 4) were mainly women. It has been noted in physiology literature that women, in general, do not empty their veins as rapidly as men [13]. Thus, it may be that when socket pressures are released (e.g., transitioning from standing to sitting), interstitial fluid levels increase dramatically within the residual limbs of women. Because the number of female subjects in the study was small and our study was not designed to test whether TRANSITION values were higher for women, we can only hypothesize this explanation. It is noted, however, that there were three other females in the study who did not show high fluid volume increases during TRANSITION. 
But all three of those subjects had PAD. Possibly these women's arterial occlusion offset their limited venous outflow so as to restrict fluid volume changes during TRANSITION. This hypothesis would need to be tested with an independent means to be verified.

In the present study, we did not control as part of the inclusion criteria prosthesis design variables such as socket type, liner material, suspension, and sock-ply. Instead we required for inclusion a properly fit prosthesis and thus an appropriate socket type, liner material, suspension, and sock-ply for each individual participant as deemed by the research practitioner. We ensured these variables were not changed within a data collection session. If they had been changed to the point that socket fit was affected, it is almost certain that limb fluid volume would have been affected. In prior investigations, adding and removing a sock affected limb fluid volume [5] and changing vacuum level affected fluid volumes during walking of elevated vacuum users [14]. The result from the present study that subjects grouped based on their REST/STAND/WALK fluid volume profiles (Figure 4) begs the question-do certain socket types, liner materials, and suspensions perform better for certain groups than others? This question needs research investigation.

A needed next step in understanding fluid volume change in people with limb loss is to determine whether the rate of fluid volume change depends upon the ordering of activities. In other words, if we monitored a person over the course of a day while they underwent resting, standing, and ambulatory activities in different orders, would the total fluid volume change over time reflect sums of the times spent at each activity? If this were the case, then we might be able to predict fluid volume changes over time through a diagnostic bioimpedance test in the clinic coupled with an activities identification monitor worn continuously on or in the prosthesis.

\section{CONCLUSIONS}

During test sessions with equal intervals of resting, standing, and walking activities conducted on 24 participants with transtibial amputation, we found that standing induced the highest residual limb fluid volume loss, with an average rate of -0.4 percent $/ \mathrm{min}$. While all subjects lost limb fluid volume during standing, 16 of the 24 subjects gained fluid volume during walking. Only 15 of the 24 gained fluid volume during resting. In general, subjects who lost fluid volume during walking gained fluid volume during resting.

There was variability across subjects as to when fluid volume was lost or recovered. The differences in fluid volume response among subjects suggest that volume accommodation technologies should be matched to the fluid transport qualities of the individual prosthesis user. The results presented are preliminary and need to be tested further with larger sample sizes.

\section{ACKNOWLEDGMENTS}

\section{Author Contributions:}

Study concept and design: J. E. Sanders.

Acquisition of data: D. S. Harrison, K. J. Allyn.

Analysis and interpretation of data: J. C. Cagle, M. A. Ciol,

J. E. Sanders.

Drafting of manuscript: J. E. Sanders.

Critical revision of manuscript for important intellectual content:

J. C. Cagle, K. J. Allyn, M. A. Ciol.

Statistical analysis: M. A. Ciol, J. C. Cagle.

Obtained funding: J. E. Sanders.

Financial Disclosures: The authors have declared that no competing interests exist.

Funding/Support: This material was based on work supported by the Congressionally Directed Medical Research Program at the Department of Defense and American Reinvestment and Recovery Act funding to the National Institutes of Health (grant R01HD60585).

Additional Contributions: We gratefully acknowledge assistance in vascular data interpretation from Elaine Tsai, MD.

Institutional Review: All study procedures were approved by a University of Washington Institutional Review Board, and informed consent was obtained before any study procedures were initiated.

Participant Follow-Up: The authors do not plan to inform participants of the publication of this study. However, participants have been encouraged to check the study Web site for updated publications.

\section{REFERENCES}

1. Board WJ, Street GM, Caspers C. A comparison of transtibial amputee suction and vacuum socket conditions. Prosthet Orthot Int. 2001;25(3):202-9. [PMID:11860094] http://dx.doi.org/10.1080/03093640108726603

2. Sanders JE, Harrison DS, Allyn KJ, Myers TR. Clinical utility of in-socket residual limb volume change measurement: Case study results. Prosthet Orthot Int. 2009;33(4): 378-90. [PMID:19961297] http://dx.doi.org/10.3109/03093640903214067

3. Sanders JE, Harrison DS, Cagle JC, Myers TR, Ciol MA, Allyn KJ. Post-doffing residual limb fluid volume change in people with trans-tibial amputation. Prosthet Orthot Int. 
2012;36(4):443-49. [PMID:22588848]

http://dx.doi.org/10.1177/0309364612444752

4. Allyn KJ, Cagle JC, Phillips RH, Harrison DS, Hartley TL, Sanders JE. Maintaining limb volume: Effects of doffing between periods of activity. 38th Academy Annual Meeting and Scientific Symposium American Academy of Orthotists and Prosthetists; 2012 Mar 21-24; Atlanta, GA. Washington (DC): AAOP; 2012.

5. Sanders JE, Harrison DS, Allyn KJ, Myers TR, Ciol MA, Tsai EC. How do sock ply changes affect residual-limb fluid volume in people with transtibial amputation? J Rehabil Res Dev. 2012;49(2):241-56. [PMID:22773526] http://dx.doi.org/10.1682/JRRD.2011.02.0022

6. Adragao T, Pires A, Branco P, Castro R, Oliveira A, Nogueira C, Bordalo J, Curto JD, Prata MM. Ankle-brachial index, vascular calcifications and mortality in dialysis patients. Nephrol Dial Transplant. 2012;27(1):318-25. [PMID:21551082] http://dx.doi.org/10.1093/ndt/gfr233

7. Potier L, Abi Khalil C, Mohammedi K, Roussel R. Use and utility of ankle brachial index in patients with diabetes. Eur J Vasc Endovasc Surg. 2011;41(1):110-16. [PMID:21095144] http://dx.doi.org/10.1016/j.ejvs.2010.09.020

8. De Lorenzo A, Andreoli A, Matthie J, Withers P. Predicting body cell mass with bioimpedance by using theoretical methods: A technological review. J Appl Physiol. 1997; 82(5):1542-58. [PMID:9134904]

9. Xitron Technologies Inc. HYDRA ECF/ICF (model 4200): Bio-impedance spectrum analyzer for measuring intracellular and extracellular fluid volumes. San Diego (CA): Xitron Technologies Inc; 2001.

10. Fenech M, Jaffrin MY. Extracellular and intracellular volume variations during postural change measured by segmental and wrist-ankle bioimpedance spectroscopy. IEEE Trans Biomed Eng. 2004;51(1):166-75. [PMID:14723506] http://dx.doi.org/10.1109/TBME.2003.820338

11. Grimnes S, Martinsen OG. Bioimpedance and bioelectricity basics. 2nd ed. London (England): Academic Press; 2008.
12. Nicolaides AN; Cardiovascular Disease Educational and Research Trust; European Society of Vascular Surgery; The International Angiology Scientific Activity Congress Organization; International Union of Angiology; Union Internationale de Phlebologie at the Abbaye des Vaux de Cernay. Investigation of chronic venous insufficiency: A consensus statement (France, March 5-9, 1997). Circulation. 2000; 102(20):E126-63. [PMID:11076834] http://dx.doi.org/10.1161/01.CIR.102.20.e126

13. Lindenberger M, Länne T. Sex-related effects on venous compliance and capillary filtration in the lower limb. Am J Physiol Regul Integr Comp Physiol. 2007;292(2):R852-59. [PMID:17038441] http://dx.doi.org/10.1152/ajpregu.00394.2006

14. Sanders JE, Harrison DS, Myers TR, Allyn KJ. Effects of elevated vacuum on in-socket residual limb fluid volume: Case study results using bioimpedance analysis. J Rehabil Res Dev. 2011;48(10):1231-48. [PMID:22234667] http://dx.doi.org/10.1682/JRRD.2010.11.0219

Submitted for publication April 5, 2013. Accepted in revised form September 17, 2013.

This article and any supplemental material should be cited as follows:

Sanders JE, Cagle JC, Allen KJ, Harrison DS, Ciol MA. How do walking, standing, and resting influence transtibial amputee residual limb fluid volume? J Rehabil Res Dev. 2014;51(2):201-12.

http://dx.doi.org/10.1682/JRRD.2013.04.0085

ResearcherID/ORCID: Joan E. Sanders, PhD: E-8204-2011

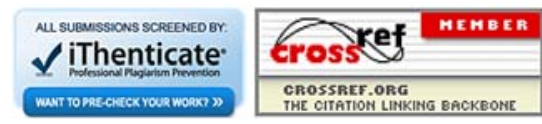

